\title{
Construction of a High Resolution Electron Beam Profile Monitor*
}

\section{$\operatorname{lng} 09$}

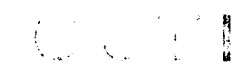

J. Norem, J. Dawson, W. Haberichter, W. Novak, L. Reed, X-F. Yang

\author{
Argonne National Laboratory \\ Argonne, IL
}

\section{DISCLAIMER}

\begin{abstract}
This report was prepared as an account of work sponsored by an agency of the United States Government. Neither the United States Government nor any agency thereof, nor any of their employees, makes any warranty, express or implied, or assumes any legal liability or responsibility for the accuracy, completeness, or usefulness of any information, apparatus, product, or process disclosed, or represents that its use would not infringe privately owned rights. Reference herein to any specific commercial product, process, or service by trade name, trademark, manufacturer, or otherwise does not necessarily constitute or imply its endorsement, recommendation, or favoring by the United States Government or any agency thereof. The views and opinions of authors expressed herein do not necessarily state or reflect those of the United States Government or any agency thereof.
\end{abstract}

\section{Presented to}

The Particle Accelerator Conference ' 93

Washington, D.C.

May $17-20,1993$

*Th1s work supported by the U.S. Department of Energy, Division of High Energy Physics, Contract W-31-109-ENG-38. 


\title{
Construction of a High Resolution Electron Beam Profile Monitor *
}

\author{
J. Norem, J. Dawson, W. Haberichter, W. Novak, L. Reed, X-F. Yang \\ Argonne National Laboratory, Argonne, IL, 60439, USA
}

\begin{abstract}
Bremsstrahlung from an electron beam on a heavy target can be used to image the beam profile using collimators and slits. The limiting resolution using this system is determined by Fresnel diffraction, and is $\sim V(\lambda d / 2)$, where $\lambda$ is the photon wavelength and $d$ is determined by the linear dimensions of the system. For linear colliders this resolution could be a few $\mathrm{nm}$. The highest resolution requires detectors which see only high energy, (small $\lambda$ ), photons, and this is accomplished by converting photons to pairs, and detecting Cherenkov light in a nearly forward angle with a CCD detector or streak camera. Tests are planned at the Argonne APS and SLAC FFTB.
\end{abstract}

\section{INTRODUCTION}

The next generation of linear colliders will require very small $(1-100 \mathrm{~nm})$ beam spots at the interaction point to produce significant luminosity, since the number of electrons which can be accelerated to high energy is limited by the available power, its cost, and the acceleration efficiency. Producing, maintaining and monitoring these spots will be be difficult. [1]

We are building a bremsstrahlung beam profile monitor which uses nonimaging optics to measure beam position and density profile, $(\rho(y)$ or $\rho(y, t))$, at the final focus of a beamline or collider[2]. The ultimate resolution is roughly $5-30 \mathrm{~nm}$, and $200 \mathrm{fsec}$, which should be sufficient to contribute to initial and final tune up, and online monitoring of beam position if active stabilization of seismic motions is required. Although the system is being developed using bremsstrahlung, backscattered photons from Compton scattering or beamstrahlung from $\mathrm{e}^{+} / \mathrm{e}^{-}$ collisions could also be used.

The system, Figure 1, consists of a Bremsstrahlung radiator at the focus of the electron beam, a single sided collimator to produce a bremsstrahlung shadow, and a slit and detector system to measure the shape of the shadow edge. The diagnostic slit could be either tilted or parallel with the primary collimator. The sharpness of the shadow is inversely proportional to the size of the s,yot at the bremsstrahlung source. Shielding, and sweeping magnets, are not shown. Finally, the bremsstrahlung photons will be detected using a pair converter and Cherenkov radiator.

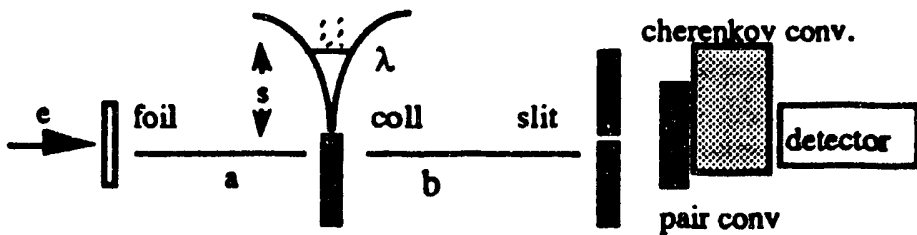

Figure 1. Bremsstrahlung radiatior, single sided collimator, slit and detector.

Work supported by the US Department of Energy, Office of High Energy and Nuclear Physics. Including Contract W-31-109-ENG-38

\section{RESOLUTION}

The ultimate resolution of this system is limited by Fresnel (circular wavefront) diffraction[3]. This limit can be approximated using Fraunhofer (plane wave) diffraction, by considering a virtual slit at the primary collimator location, where the virtual slit width, $s$, is such that the sagitta is equal to $\lambda$, the photon wavelength. If the source to collimator distance is $a$, the collimator to detector distance is $b$, and $b \gg$ $a$, the expression for the sagitta $\lambda=s^{2} / 2 a$ gives the virtual sit width, $s=\sqrt{ }(2 \lambda a)$, (see Fig 1). The angular diffraction width is then $\lambda / s$ and the limiting resolution at the foil is roughly $(\lambda / s) a$ $\sqrt{ }(\lambda \mathrm{a} / 2)$, nearly the geometric mean of the beamline dimensions, $(1-10 \mathrm{~m})$, and the photon wavelength, $\left(\lambda=10^{-16}\right.$ $\mathrm{m}$ at $10 \mathrm{GeV}$ ). Improving the resolution requires neducing $a$, by moving collimators close to the target, or $\lambda$, by detecting the highest energy photons.

Accurate calculations require: 1) Fresnel optics, which is done for monochromatic light in most optics books, and 2) a complete photon spectrum which includes the actual detector acceptance function. The relevant spectra are bremsstrahlung, with $n_{y}(y) \equiv 1 / y$, Compton scattering, with $n_{y}(y) \equiv$ const, and $n y(y) \equiv y^{-2 / 3}(1-y)^{1 / 3} e^{x y /(1-y)}$, from beamstrahlung at the IP. Here $y=E_{\gamma} / E_{e, \max }$ and $k=2 / 3 Y$, and $Y$ is the fractional energy lost to individual photons.[4] The acceptance of the detector has been evaluated using EGS4 and and a more specialized monte carlo program which generates a bremsstrahlung spectrum, computes pair production and subsequently evaluates multiple scattering. The brems spectrum are shown in ref [2]. Integrating Fresnel diffraction patterns for the appropriate energy spectra gives the complete profile. This note defines a resolution function as the derivative of this sum of diffraction images, shown in Fig 2, This curve, which is nongaussian, is the effective shape of a beam at the bremsstrahlung radiator in the limit of a zero width slit at the detector.

An option for the bremsstrahlung detector is shown in Fig 1 , with pair converter followed by Cherenkov radiator. Sweeping magnets may be required to reduce shower background. The number of Cherenkov photons detected should be on the order of 2000 / half width. which stould be sufficient for the operation of a streak camera. Time resolution on the order of $\sigma=200 \mathrm{fsec}$ should be obtainable with the Hamamatsu FESCA 500 streak camera[5], which should be able to examine structure within individual bunches. This resolution is only available with slit widths of $10 \mu \mathrm{m}$, which will be difficult.

Ionization of the bremsstrahlung radiator, motion of the target ions, multiple scattering, depth of focus, beam focusing by the plasma created in the radiator, and synchrotron radiation 
by the primary electrons, should be detectable, and correctable, effects Multiple scattering and focusing should be small effects compared to the divergence of the primary beam. Synchrotron radiation should be detectable, at colliders, with fractional photon energy $Y=0.1$, but perhaps also at the FFTB as well, where $Y=0.002$. Ions will be focused by the beam, increasing the local density of the bremsstrahlung radiator, although this should be correctable.
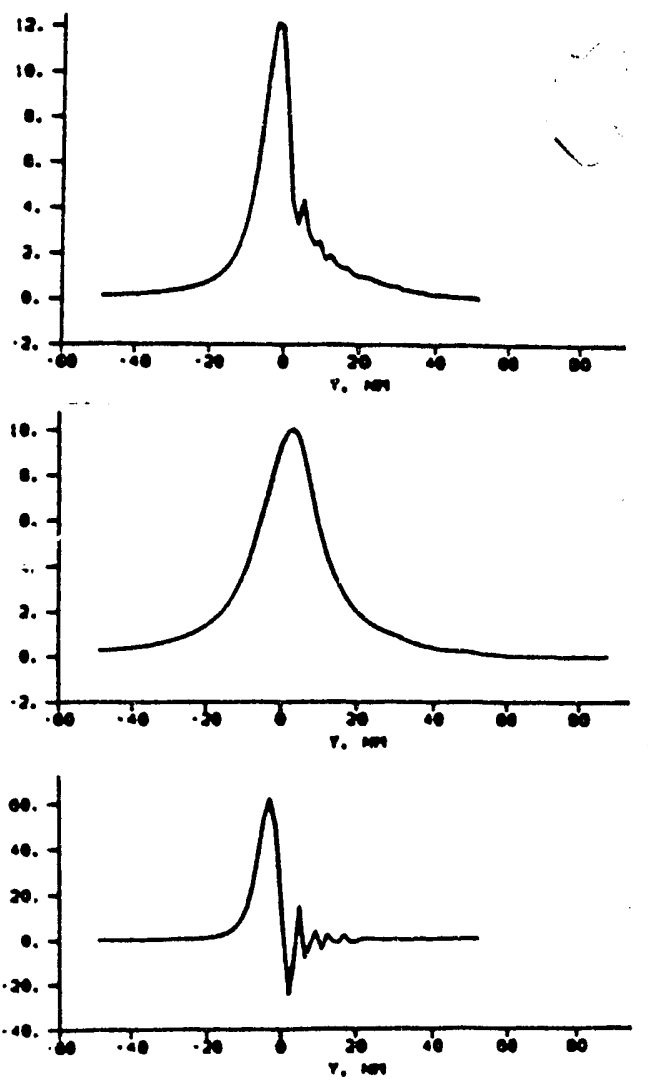

Figures 2 Resolution functions for bremsstrahlung (top), beamstrahlung (middle) and Compton scattering (bottom) using possible linear collider conditions, $\quad E_{e}=250 \mathrm{GeV}$, a $=10 \mathrm{~m}, \mathrm{~b}=200 \mathrm{~m}, \theta=2^{\circ}$ ). All are nongausian

The thick foil in the beam will be a source of background in the experiment as well as an additional constraint on the shielding. In principle, since the emittance of the signal is so small, it should be possible to produce a reasonable signal /noise ratio. by moving detectors downstream to a more quiet environment.

\section{COMPONENTS}

The bremsstrahlung radiator will be thin, $0.1 \mathrm{~mm}$ foils. Possible materials would include Ta, W, Ph, U, and Au, all of which are sold in many thicknesses and sizes by Goodfellow Corporation[6]. Tungsten and tantalum have the highest heat of vaporization. which seems to be the best measure of their stability, but platinum, uranium or gold foils could also be useful. These materials would be locally destroyed on every pulse. Energy deposited in the foil due to $d E / d x$ losses would be on the order of $0.6 \mathrm{~mJ} / \mathrm{pulse}$. This energy would be sufficient to vaporize a hole $10 \mu \mathrm{m}$ in radius in a tungsten foil. The foils would be moved after every pulse.

The collimators and slit edges have to be flat, and thick enough to stop the beam. Tungsten mirrors can be produced which are $3^{n}$ in diameter, and optically flat, $\lambda / 20=$ $(\sigma \approx 10 \mathrm{~nm})$, for reasonable prices, with better surface quality possible at higher prices[7]. With a radiation length of about $0.31 \mathrm{~cm}$, direct photons would be attenuated by $10^{-11}$ if they penetrate the collimator. Encodermotors or stepping motors are both sold which have setting errors of about $\sigma=30 \mathrm{~nm}$, which should be sufficient. Finer motions can be achieved with peizomovers. Guard collimators, located in front of the primary collimator and slit, should be cooled.

The camera, shown in Figure 3 uses a Hamamatsu V4183U two stage, gated image intensifier which has single photon sensitivity, mechanically in contact with a Hamamatsu \$3902 MOS linear image sensor[5]. Fiber optic exit and entrance windows permit high resolution. Measurements of single photons have shown that the resolution is very good, $0=25$ $\mu \mathrm{m}$, depending somewhat on pulse height. The radiation sensitivity of this system has heen tested and it should operate well in a $100 \mathrm{mR} / \mathrm{hr}$ envionment. The manufacturer claims that the components should not be particularly sensitive to radiation damage.

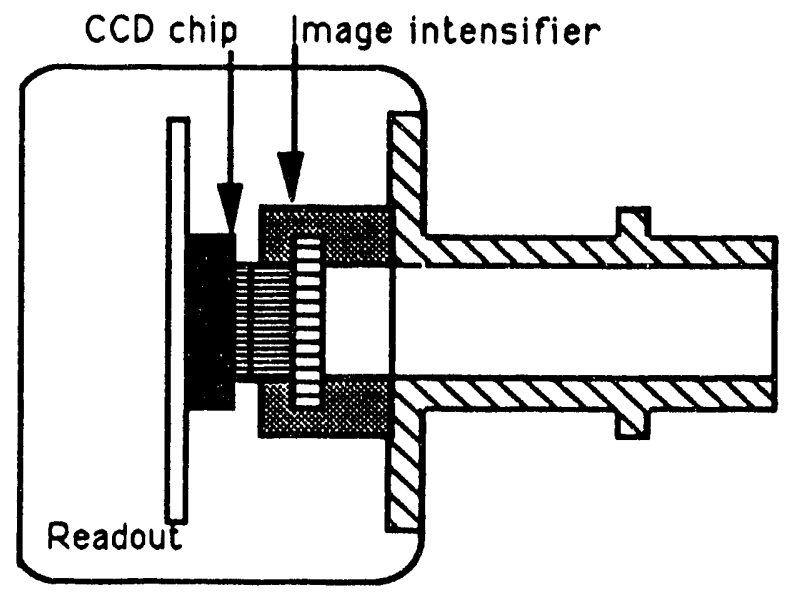

Figure 3. The camera.

The camera controller is a single width CAMAC module containing the circuitry for control and readout the CCD camera. Event In, a NIM level input, starts the cycle to read in the CCD. The video signal from the camera is buffered to an 8-bit analog to digital converter (ADC), which writes its output data into a 4K by 8-bit First In First Out (FIFO) memory. This memory can be written or read by the computer thru the CAMAC dataway. A crystal oscillator generates a clock $(312.5 \mathrm{KHz})$ for the $C C D$ readout and provides two other frequencies, $9.8 \mathrm{KHz}$ and $76 \mathrm{KHz}$, which will be used for seismic sensing. An 8-bit status register keeps track of status conditions in the module as well as the condition of two NIM inputs. The status register can also be written or read from the computer.

The control computer, a 486 clone, uses LabWindows[8], and the code is written in C. It presently controls 8 encoder 
motors, the printer and the camera. It will also control the seismic correction system and Ethernet communications when needed.

The collimators can be roughly aligned using transets and levels to about $100 \mu \mathrm{m}$ It is assumed that the slits can be opened and bremsstrahlung signals can be used to align the system from this point. For high precision measurements, seismic motions at the $50-150 \mathrm{~nm}$ level must be corrected. We plan to have a seismic correction system which can compensate these motions, and have ordered a Streckeisen STS-2 seismometer[9] which is very sensitive in the frequency range $0.1-10 \mathrm{~Hz}$. The measured noise level of this device is two orders of magnitude below midcontinent nighttime ambient earth noise. Corrections could be made either on line with piezomovers, or off line by correcting the data.

\section{TESTS}

The Argonne Advanced Photon Source (APS) is scheduled to be completed in 1995, and the electron/positron linac injector should be running in the summer of 1993. Parasitic bremsstrahlung from the positron production target can provide a useful test of the proposed system, because the electron beam energy is high enough to be detected (200 $\mathrm{MeV}$ ), and high enough to produce a realistic shower background, and there seems to be space enough downstream of the positron linac to mount collimators, slits and detectors.

A more challanging test of the system would be to measure the properties of the $\sigma=60 \mathrm{~nm}$ beam spot at the SLAC/FFTB. The primary collimator would be located about 20 feet downstream of the dump line bending magnets and 28 $\mathrm{m}$ from the IP. In front of the primary single sided collimator would be a guard collimator system, consisting of two tungsten or tantalum blocks ground flat and spaced apart by $0.00025^{\prime \prime}-0.001^{\prime \prime}$ with shims. The guard collimator would serve two purposes: minimizing beam heating and deflection of the primary collimator, and providing a "black" background against which the beam on target can be seen.

This system should permit measurements of the beam profile, $\rho(x, t)$, at the final focus, which might be used to study single or multibunch beam instabilities driven by wake fields, and possible nonlinearities due to beam optics. The high resolution should permit study of a variety of other effects, such as the Oide limit; where the $\sigma \propto \varepsilon^{5 / 7}[1]$

The technique should also be useful for plasma lens experiments where focusing is inherently nonlinear, due to inhomogenities in the transverse and longitudinal charge density, plasma response time, $1 / \omega_{\mathrm{p}}$, and uneven plasma ionization. Deconvolving beam shape and beam jitter also make single bunch measurements desirable.

\section{CONCLUSIONS}

Nonimaging optics should provide useful information about beam shapes at high energy electron accelerators and collidars. Bremsstrahlung is the easiest source of high energy photons, however beamstrahlung in colliders should provide a passive method of looking at $e^{f} / e^{-}$collisions, and Compton backscatters could be used to produce higher energy photons and better resolution for single beam measurements.

We are building a system qhich should make it possible to study the technique experimentally at the Argonne APS and SLAC FFTB.

\section{REFERENCES}

[1] R. B. Palmer, Annu. Rev. Nucl. Part. Sci. 40, 529, (90)

[2] J. Norem, Rev. Sci. Instrum., 62(6), (1991), 1464

[3] Jenkins and White, Fundamentals of Optics, Ch 18, Fresnel Diffraction, McGraw-Hill (1957)

[4] P. Chen, P., Phys. Rev. D, 46, (1992), 1186.

[5] Hamamatsu Photonic Systems, Bridgewater, NJ, 08807

[6] Goodfellow Corporation, Malvern, PA 19355

[7] R Lowrey, Rockwell Power Systems, Albuquerque NM (private communication)

[8] National Instruments, Austin, TX, 78730

[9] Quanterra, Inc. Harvard, MA, 01464 

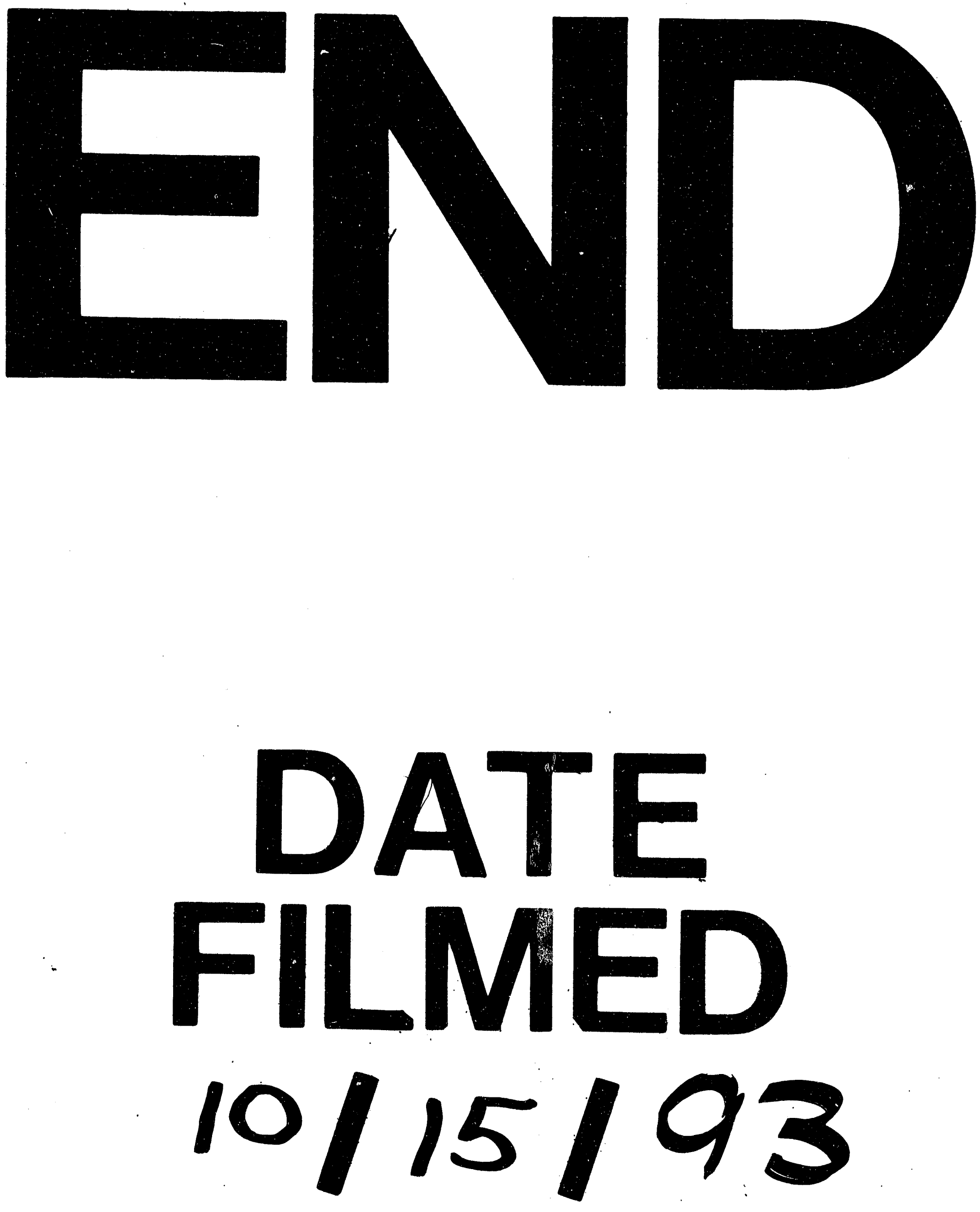
